Article type : Articles

Running head: Coexistence and niche shifts

\title{
PREDICTING COEXISTENCE IN SPECIES WITH CONTINUOUS ONTOGENETIC NICHE SHIFTS AND COMPETITIVE ASYMMETRY
}

\author{
Ronald D. Bassar ${ }^{1, \dagger}$, Joseph Travis ${ }^{2}$, and Tim Coulson ${ }^{3}$ \\ ${ }^{1}$ Department of Biology, Williams College, Massachusetts, USA \\ ${ }^{2}$ Department of Biological Science, Florida State University, Florida, USA \\ ${ }^{3}$ Department of Zoology, University of Oxford, Oxford, UK \\ ${ }^{\dagger}$ Corresponding author - email: ronald.d.bassar@williams.edu
}

\section{ABSTRACT}

A longstanding problem in ecology is whether structured life cycles impede or facilitate coexistence between species. Theory based on populations with only two discrete stages in the life-cycle indicates that for two species to coexist, at least one must shift its niche between stages and each species must be a better competitor in one of the niches. However, in many cases, niche shifts are associated with changes in an underlying continuous trait like body size and we have few predictions concerning conditions for coexistence for such a widespread form of ontogenetic development. We develop a framework for analyzing species coexistence based on Integral Projection Models (IPMs) that incorporates continuous ontogenetic changes in both the resource niche and competitive ability. We parameterize the model using experimental data from Trinidadian guppies and show how niche shifts and competitive symmetries impact species coexistence. Overall, our results show that the effects This article has been accepted for publication and undergone full peer review but has not been through the copyediting, typesetting, pagination and proofreading process, which may lead to differences between this version and the Version of Record. Please cite this article as doi: 10.1002/ecy.1969

This article is protected by copyright. All rights reserved. 
of competition on fitness depend upon trait-mediated niche-separation, trait-mediated competitive asymmetry in the part of the niche that is shared across body sizes, and the sensitivity of fitness to body size. Interactions among these processes generate multiple routes to coexistence. We discuss how our modelling framework expands results from twostage models to mutli-stage or continuous stage models and allows for deriving predictions that can be tested in populations displaying continuous changes in niche use and competitive ability.

Keywords: Coexistence, Alternate stable states, Integral projection models, Competition, Ontogenetic niche shifts, Asymmetric competition

\section{INTRODUCTION}

For many organisms, resource use patterns change throughout development (Werner and Gilliam 1984). Such ontogenetic niche shifts range from separate niches in discrete life stages as seen in amphibians or aquatic insects, to continuous changes in niche use as a function of a trait that changes during development (e.g. body size in insects, fishes, mammals, and reptiles). Some organisms exhibit both discrete and continuous niche shifts. For example, dragonflies occupy discrete niches with aquatic naiads and terrestrial adults, but the food habits of the naiads change dramatically as size and instar number increases (Corbet 1980). Such ontogenetic changes lead to the structuring of populations into both discrete and continuous stages with the strength of the competition determined by both the stage and size of the focal individual and the competitor.

One persistent question associated with such structured life cycles is whether they promote or impede species coexistence (Miller and Rudolf 2011, Nakazawa 2015). In simple, two-species models with two discrete life stages (e.g. larvae and adults), coexistence of two 
species depends on the nature of change in the niche with stage, the asymmetry in the outcome of competition between the species at different stages, and which stage primarily limits the growth of each species (Haefner and Edson 1984, Loreau and Ebenhoh 1994, Moll et al. 2008, Ackleh and Chiquet 2011, de Roos and Persson 2013). For example, when one species shifts its niche with stage and the other does not, coexistence is possible if the one that shifts its niche is primarily limited in the stage that escapes competition with the other species; otherwise the species that shifts its niche will exclude the other species (Loreau and Ebenhoh 1994). Competitive asymmetries can alter these predictions; for example, if the species that does not shift is a much better competitor, it will competitively exclude the species that does shift its niche (Loreau and Ebenhoh 1994).

While predictions like these apply in many cases, for a considerable number of species, life stages cannot be categorized into discrete stages. For these species, the life history, changes in the niche, and competitive ability are often more accurately described by a continuous trait (Werner and Gilliam 1984, Reñones et al. 2002, Briones et al. 2012). For a wide variety of organisms, the trait that determines the niche and competitive ability is body size (Werner and Gilliam 1984, Reñones et al. 2002, Wallace and Leslie 2008, Briones et al. 2012). Organisms with continuous changes in body size display a wide variety of life histories and changes in competitive abilities through development, many of which are difficult to capture by collapsing the life history into two life stages (Easterling et al. 2000). For these species, no single stage limits the growth of such populations; population limitation is instead spread across the spectrum of sizes in the population, often in complex ways (Bassar et al. 2013). 
Applying two-stage theory to such populations can distort the conceptual issues and create practical challenges. On the conceptual side, taking this approach requires constructing size classes on either side of an important transition in the life cycle, such as size at maturation. In doing so, one is forced to assume that the transition occurs at fixed sizes that do not change with resource acquisition, which is uncharacteristic of most continuously growing organisms. This approach can also overlook the role played by the duration of each stage, a role more easily captured for size-dependent niches and competitive ability by explicitly incorporating somatic growth rate. On the practical side, if there is no obvious transition in the life cycle demarcating size classes, as would be the case for continuously growing organisms, testing these models requires an arbitrarily delineation of size classes, which means that the results could be sensitive to how size classes are defined (Easterling et al. 2000). It is thus unclear how coexistence theory built on niche shifts between discrete life stage translates into robust, testable predictions of coexistence for organisms with continuous life stages (Nakazawa 2015).

Here, we develop a framework for predicting coexistence in discrete time models with continuous traits that determine ontogenetic changes in niche use and competitive asymmetry. We develop a single-sex, two-species Integral Projection Model (IPM) where each species has identical underlying demography and can shift its niche to varying degrees with development. We incorporate into our models asymmetries in competition that depend on body size, and ask how linear ontogenetic niche shifts and changes in competitive ability with body size lead to species coexistence or competitive exclusion. We provide the groundwork for understanding how conditions for coexistence can be analyzed in these models using invasion analysis. We then generate predictions for the model under three ecological scenarios that are analogous to previous work with discrete stage models and show 
how transitions between competitive exclusion and coexistence arise as a balance between stabilizing and destabilizing mechanisms across the spectrum of sizes in the populations. We discuss how this model confirms and extends predictions about coexistence compared to previous models of coexistence in structured populations, and how it can be applied to data from natural and experimental populations.

\section{MATERIALS AND METHODS}

\section{MODELLING FRAMEWORK}

We follow the structured modelling approach developed in our previous work on competitive asymmetries based on Integral Projection Models (IPMs) (Bassar et al. 2016). A simple single species, single sex (female only) IPM is written:

$$
n\left(z^{\prime}, t+1\right)=\int_{L}^{U}\left[G\left(z^{\prime} \mid z\right) S(z)+D\left(z^{\prime} \mid z\right) M(z) B(z) S(z)\right] n(z, t) d z .
$$

The function $n(z, t)$ describes the density of individuals in the population at time $t$ across all $z$ trait values such that $N=\int_{L}^{U} n(z, t) d z$ is the total density of individuals in the population at time t. $L$ and $U$ are the lower and upper limits of the trait values. Here we assume $z$ is body length, but the model can be used to describe any continuous trait that changes with ontogeny. $S(z)$ and $B(z)$ are functions respectively describing the probability of an individual with body size $z$ surviving the time interval and reproducing at the end of the interval. $M(z)$ is a function describing the expected litter size of an individual with body size z. $G\left(z^{\prime} \mid z\right)$ describes the probability of a surviving individual with body size $z$ at time $t$ growing to body size $z^{\prime}$ at time $t+1 . D\left(z^{\prime} \mid z\right)$ describes the probability that a parent with body size $z$ at time $t$ produces an offspring with trait value $z^{\prime}$ at time $t+1$. The structure of the model assumes that individuals give birth to offspring immediately preceding the next census.

This article is protected by copyright. All rights reserved. 
The relationship between size and each of these vital rates can be estimated using generalized linear models (Easterling et al. 2000, Coulson et al. 2010, Coulson 2012, Rees et al. 2014, Bassar et al. 2016). For example, the probability of surviving the interval can be estimated using a generalized linear model with a binomial error structure and logit link function:

$$
\operatorname{logit}(S(z)) \sim V(z)
$$

Equations for each of the vital rates are given in Table 1.

Bassar et al. (2016) showed that one way to incorporate density- and frequencydependence is to make the function describing any appropriately transformed vital rate, $V$, a function of body size and the number and distribution of body sizes (or other traits) in the population as:

$$
V(z, N, p)=\beta_{0}+\beta_{z} z+\beta_{N} N \int_{L}^{U} \alpha(z, x) p(x) d x
$$

Here $z$ represents the trait value of individuals whose vital rates are being measured and $x$ represents the trait values of competitors in the population. Importantly, $z$ and $x$ represent the same trait. $p(x)=n(x) / N$ is the frequency of individuals with trait $x$. The terms, $\beta_{0}+\beta_{z} z$, describe the expected value of the vital rate when $N=0$ (i.e. the density-independent terms) and how it changes as a function of body size, $z$. The parameter $\beta_{N}$ describes how the vital rate is affected by a change in the density of the population, independent of the trait values in the population. When density decreases the vital rate, as in competitive interactions, $\beta_{N}$ is negative. The function $\alpha(z, x)$ defines an interaction surface. It describes the strength of competitive interactions, measured as the number of individuals with trait value $x$ that are equivalent in competitive ability to an individual with trait value $z$ (Bassar et al. 2016). Bassar et al. (2016) describes how asymmetric competition between sizes in single species models alters the vital rates, population dynamics, and evolutionary quantities such as 
generation time and provides alternative formulations of the vital rate equations that are not additive.

Extending the model to more than one species involves adding an additional term to the vital rate equations that describes the demographic impact of the other species. If $V_{i}$ is the vital rate equation for species $i, z_{i}$ is the body size of a focal individual of species $i$, and $x_{j}$ represents the body sizes of species $j$, then our equation for the vital rate of species $i$ is:

$$
V_{i}\left(z_{i}, N_{i}, N_{j}, p_{i}, p_{j}\right)=\beta_{i, 0}+\beta_{z_{i}} z_{i}+\beta_{i, N} N_{i} \int_{L_{i}}^{U_{i}} \alpha_{i i}\left(z_{i}, x_{i}\right) p\left(x_{i}, t\right) d x_{i}+\beta_{i, N} N_{j} \int_{L_{j}}^{U_{j}} \alpha_{i j}\left(z_{i}, x_{j}\right) p\left(x_{j}, t\right) d x_{j} . \quad 4
$$

The interaction surface $\alpha_{i i}\left(z_{i}, x_{i}\right)$ describes the within species equivalence of an individual of with body size $x$ on an individual with body size $z$. The interaction surface $\alpha_{i j}\left(z_{i}, x_{j}\right)$ describes the between species equivalence of an individual of species $j$ with body size $x$ on an individual of species $i$ with body size $z$.

The interaction surface can have many forms, depending on how niches are partitioned and how competitive ability changes with body size. Bassar et al. (2016) describe several possible forms when competitive asymmetries among individuals depend on body size, but where all individuals are assumed to compete for the same resources (i.e. they have the same niche). Interaction surfaces that incorporate ontogenetic niche shifts, competitive asymmetries between species, and competitive asymmetries between body sizes have a different form. Following Macarthur and Levins (1967), we assume that the distribution of resource utilization of species $i$ can be described by the equation $e^{-\frac{\left(\mu\left(z_{i}\right)-R\right)^{2}}{H^{2}}}$, where $\mu\left(z_{i}\right)$ is the mean position of the resource use distribution on a linearized resource axis, $R$, and $H^{2}$ is the niche width, which we assume to be equal to 1 for all sizes and species. Here we assume the mean niche position is a linear function of body size such that $\mu\left(z_{i}\right)=\iota_{i}+\rho_{i}\left(z_{i}-\omega\right)$. The parameter $\iota_{i}$ defines the mean niche position of body size $\omega$ in species $i$ and $\rho_{i}$ describes how the mean niche position changes with body size in species $i$. We then assume that the 
area under the niche function can be interpreted as the total resource utilization and can be scaled by a linear function of body size on the natural $\log$ scale: $e^{\eta_{i}+\phi_{i}\left(z_{i}-v\right)}$, where the parameter $\eta_{i}$ defines the resource use of body size $v$ in species $i$ and $\phi_{i}$ describes how resource acquisition changes with body size in species $i$. Combining these functions yields: $U_{i}\left(R, z_{i}\right)=e^{\eta_{i}+\phi_{i}\left(z_{i}-v\right)} e^{-\frac{\left(\iota_{i}+\rho_{i}\left(z_{i}-\omega\right)-R\right)^{2}}{H^{2}}}$. In keeping with the standard interpretation of interaction coefficients as relative effects, the interaction surface, or the amount of niche overlap scaled by competitive asymmetry between species is then calculated as:

$$
\begin{aligned}
\alpha_{i j}\left(z_{i}, x_{j}\right) & =\frac{\int U_{i}\left(R, z_{i}\right) U_{j}\left(R, x_{j}\right) d R}{\int\left[U_{i}\left(R, z_{i}\right)\right]^{2} d R} \\
& =\frac{e^{\eta_{i}+\phi_{i}\left(z_{i}-v\right)} e^{\eta_{j}+\phi_{j}\left(x_{j}-v\right)}}{\left(e^{\left.\eta_{i}+\phi_{i}\left(z_{i}-v\right)\right)^{2}}\right.} \frac{\int e^{-\frac{\left(\iota_{i}+\rho_{i}\left(z_{i}-\omega\right)-R\right)^{2}}{H^{2}}} e^{-\frac{\left(\iota_{j}+\rho_{j}\left(x_{j}-\omega\right)-R\right)^{2}}{H^{2}}} d R}{\int\left(e^{-\frac{\left(\iota_{i}+\rho_{i}\left(z_{i}-\omega\right)-R\right)^{2}}{H^{2}}}\right)^{2} d R} \\
& =e^{\eta_{i j}+\phi_{z, j}\left(x_{j}-v\right)-\phi_{z, i}\left(z_{i}-v\right)} e^{-\frac{\left(\iota_{i j}+\rho_{j}\left(x_{j}-\omega\right)-\rho_{i}\left(z_{i}-\omega\right)\right)^{2}}{2 H^{2}}}
\end{aligned}
$$

where $\iota_{i j}$ is the difference in the mean niche positions between species at size $\omega$ and $\eta_{i j}$ measures the difference in resource use between the species at size $v$.

Each of the parameters of the interaction surface alters the shape of the interaction surface in different ways (Figure 1). When species $i$ does not shift its niche with size $\left(\rho_{i}=\right.$ 0 ), species $j$ shifts its niche with size $\left(\rho_{j}=0.15\right)$, and all individuals of both species are equal competitors (symmetric interaction; $\eta_{i j}=0, \phi_{i}=0, \phi_{j}=0$ ), then the intraspecific interaction surface for species $i$ is flat (row 1, column 1 of Figure 1). In contrast, the interspecific interaction surface decreases with increasing competitor size (row 1, column 2 of Figure 1). For species $j$, the interspecific interaction surface (row 1, column 3 of Figure 1) decreases with increasing size of individuals of species $i$. The intraspecific interaction surface of species $j$ leads to a situation where individuals of species $j$ compete most strongly with 
individuals of the same size (row 1, column 4 of Figure 1). Increasing the competitive advantage of larger individuals of species $i$, then distorts the shape of all interaction surfaces involving species $i$ such that larger individuals of species $i$ have greater effects on competition (row 2 of Figure 1).

\section{MODEL PARAMETERIZATION AND ECOLOGICAL SCENARIOS}

\section{Model parameterization}

We parameterized the model using demographic data from Trinidadian guppies (Poecilia reticulata) to facilitate analysis using reasonable parameter values. Guppies are small stream fish that inhabit freshwater streams on the Caribbean island of Trinidad. In our analysis, we assume both species (hereafter species 1 and 2) in the model have an identical guppy life history - the $\beta$ parameters in equation 4 are the same between the species. In general, any life history differences between the species should also influence the model predictions, but such an analysis is beyond the scope of this initial analysis.

The model is parameterized from the results of experiments in mesocosms and in natural streams. Briefly, each of these experiments used guppies from streams where they and Hart's killifish (Rivulus hartii) are the only fish species (i.e. the so-called "low predation" guppy life history). In the mesocosm experiments, guppies were added at two densities. In the real stream experiments, natural populations were either decreased by one-half or kept at ambient densities. The duration of each experiment was 28 days, which is slightly longer than one reproductive cycle of guppies. At the end of the mesocosm experiments, guppies were removed from the mesocosms and measured for somatic growth (mm standard length, hereafter SL), reproductive status (pregnant or not pregnant), fecundity, and offspring size (mm SL). Similar measurements were taken from the fish in the experiments in natural streams, but we only use data on survival here. More details of the experiments are described 
in detail in Bassar et al. (2013). The parameters were estimated using general linear mixed or generalized linear mixed models assuming guppies do not shift their niche with size $(\rho=0)$ and that competition among sizes is symmetrical $(\phi=0)$ (see Appendix S1: Table S1 for estimated parameters). As in Bassar et al. (2016), we did not attempt to fit values for the $\rho$ or $\phi$ parameters because this requires more detailed experiments than are currently available. The interval over which the IPM projects population dynamics is 28 days. The size interval over which the projection is calculated is $2 \mathrm{~mm}$ to $35 \mathrm{~mm}$ standard length (SL). Although guppies are never smaller than $5 \mathrm{~mm}$ at birth, decreasing the lower limit prevents any loss of individuals from the modelled population (Williams et al. 2012). Likewise, wild guppies from these populations rarely obtain $30 \mathrm{~mm}$ in size and the extreme upper limit prevents the unintentional loss of adults from the population (Williams et al. 2012).

\section{Ecological scenarios}

In scenario 1, we assumed that individuals of both species (species 1 and 2) shift their niche to varying degrees with increases in body size $\left(\rho_{1} \geq 0, \rho_{2} \geq 0\right)$ and that there were no species or size based competitive asymmetries $\left(\eta_{12}=0, \phi_{1}=0, \phi_{2}=0\right)$. For this and all other scenarios, we assumed that there were no general differences in the niches between species $\left(l_{12}=0\right)$. We initiated these scenarios using niche shift $(\rho)$ values for both species that range from 0 (no ontogenetic shift) to 0.17 , a value at which the largest individuals do not compete with the smallest individuals. We chose to use $v=6.5$ and $\omega=6.5$ so that in the case of no ontogenetic niche shifts of species 1 , all individuals of species 1 compete most strongly with newborns of species 2 (which are $\sim 6.5 \mathrm{~mm}$ ) and vice versa.

In scenario 2 we modelled an ecological situation where the competitive ability of individuals of species 1 increased with increasing body size and, as in scenario 1, each species could shift its niche to varying degrees. The increases in competitive ability of larger 
individuals of species 1 were modelled by changing $\phi_{1}$ from 0 to 0.02 . When $\phi_{1}=0.02$, a $20 \mathrm{~mm}$ individual of species 1 has a competitive advantage over a $10 \mathrm{~mm}$ individual of species 1 of about $22 \%$ and all individuals of species 2 of about $49 \%$. As in scenario 1, we held $v=6.5$ and $\omega=6.5$. The consequence of this decision was that mean size newborns of both species occupied the same niche and were equally competitive. Competitive ability of species 2 remained the same throughout life, but competitive ability of species 1 increased with increasing size.

In scenario 3, we modelled an ecological situation where larger individuals of species 1 are better competitors and smaller individuals of species 2 are better competitors and, as in scenarios 1 and 2, each species could shift its niche to varying degrees. We changed $\phi_{1}$ from 0 to 0.06 and $\phi_{2}$ from 0 to -0.06 . We set $v=15$ and $\omega=6.5$. The consequence of these parameter values is that below $15 \mathrm{~mm}$, individuals of species 1 are worse competitors than individuals of the same size of species 2 , but above $15 \mathrm{~mm}$ individuals of species 1 are better competitors than individuals of species 2 of the same size. Overall, this last scenario represents the case where each species is a better competitor at different life stages.

For each of the ecological scenarios, the parameters related to competitive asymmetry $\left(\eta_{12}, \phi_{1}, \phi_{2}\right)$ or ontogenetic niche shifts $\left(\iota_{12}, \rho_{1}, \rho_{2}\right)$ were varied in all of the demographic rates. Thus, the scenarios do not represent scenarios where changes in competitive asymmetry influences, for example, somatic growth rate but not the other demographic rates.

\section{MODEL ANALYSIS}

Coexistence means that both species can occupy a habitat despite, or perhaps because of, interspecific interactions. One way to ask whether coexistence between two species is possible is to examine the invasibility of each species at the single species equilibrium (e.g.

where $N_{i}=0$ and $N_{j}=\widetilde{N}_{j}$ ). Assuming that each species supports a single species equilibrium 
at which its' abundance is non-zero (i.e. the resident species), the ability of the missing species (i.e. the invader) to invade is determined by the stability of the zero equilibrium. When the equilibrium is stable, invasion cannot occur and the invader population size should return to 0 when perturbed. When the zero equilibrium of the invader is unstable, the invading population can grow in numbers when perturbed above zero (Roth et al. In press). Coexistence is predicted to occur when the zero equilibria of both species are unstable when evaluated at the single species equilibrium of the other species. This general approach is valid for a wide range of complex population and community dynamics including those that are chaotic (Roth et al. In press). For the scenarios modelled here, the single species equilibrium distributions were stable across the range of niche shift and size-dependent competitive asymmetries (Appendix S1: Figure S1).

General methods for stability analysis of structured demographic models are given in Caswell (2001). Briefly, the stability of the zero equilibrium is evaluated using the typical tools of a stability analysis, which involve making small perturbations to the invader population size and evaluating its growth rate. If species $i$ is the invader and species $j$ the resident, then the first-order matrix approximation of this perturbation for a discretized IPM at the zero equilibrium of the invader $\left(\mathbf{n}_{i}=0\right)$ and the single species equilibrium of the resident $\left(\mathbf{n}_{j}=\widetilde{\mathbf{n}}_{j}\right)$ is:

$$
\boldsymbol{\varepsilon}_{i}(t+1)=\left.\mathbf{K}_{i}\right|_{\mathbf{n}_{i}=0, \mathbf{n}_{j}=\widetilde{\mathbf{n}}_{j}} \boldsymbol{\varepsilon}_{i}(t),
$$

where $\boldsymbol{\varepsilon}_{i}(t)$ is a vector containing values that represent the density of invading individuals of species $i$ and $\left.\mathbf{K}_{i}\right|_{\mathbf{n}_{i}=0, \mathbf{n}_{j}=\widetilde{\mathbf{n}}_{j}}$ is the projection matrix approximation of the continuous projection kernel. It is a square projection matrix with row elements denoted $r$ and column elements denoted $s$. It is evaluated at the zero equilibrium of species $i$ and the positive single species equilibrium of species $j$. Stability of the zero equilibrium of species $i$ is then given by the dominant eigenvalue of the matrix $\left.\mathbf{K}_{i}\right|_{\mathbf{n}_{i}=0, \mathbf{n}_{j}=\widetilde{\mathbf{n}}_{j}}$, hereafter noted $\lambda_{i n v_{i}}$. The zero 
equilibrium is stable if $\lambda_{i n v_{i}}<1$ and unstable if $\lambda_{i n v_{i}}>1$. Coexistence then requires mutual invasibility between the species. In other words, species $i$ can invade species $j\left(\lambda_{i n v_{i}}>1\right)$ and species $j$ can invade species $i\left(\lambda_{i n v_{j}}>1\right)$. If species $j$ competitively excludes species $i$, then $\left(\lambda_{i n v_{i}}<1\right)$ and $\left(\lambda_{i n v_{j}}>1\right)$ and vice-versa if species $i$ can competitively exclude species $j$. In the case where neither species can invade the other $\left(\lambda_{i n v_{i}}<1\right.$ and $\left.\lambda_{i n v_{j}}<1\right)$, then this implies bistability, with the outcome depending on the initial conditions.

For each of the ecological scenarios, we present figures that show combinations of niche shifts of both species that result in competitive exclusion, coexistence and bistability. These plots were created by calculating $\lambda_{i n v}$ for each species across a range of niche shifts ( $\rho=0$ to $\rho=0.17$ ). We then show how $\lambda_{\text {inv }}$ for each species changes assuming species 1 does not shift its niche and species 2 does to illustrate how changes in the niche shift of a single species influences $\lambda_{i n v}$ of both species. Next, we calculated the niche shift values where $\lambda_{\text {inv }}$ transitions from being stable to unstable, or vice-versa. We calculated these values of the parameters using numerical iteration. In scenario 3, we additionally calculated these boundaries assuming that both species shift their niches equally with size. This was done so that we could capture the boundary of the priority effects predicted in this scenario. At each of these points, we then calculated the contribution of each size in the invading population to $\lambda_{i n v}$. The contributions of each size to $\lambda_{i n v_{i}}$ were calculated as $\mathbf{v}_{i}^{\mathrm{T} \circ}\left(\left.\mathbf{K}_{i}\right|_{\mathbf{n}_{i}=0, \mathbf{n}_{j}=\widetilde{\mathbf{n}}_{j}} \mathbf{p}_{i}\right)^{\mathrm{T}}$, where ${ }^{\circ}$ represents the Hadamard, or element-by-element, product, $\mathbf{p}_{i}$ is a right eigenvector corresponding to $\lambda_{i n v_{i j}}, \mathbf{v}_{i}{ }^{\mathrm{T}}$ is the corresponding left eigenvector, and the eigenvectors are scaled so that the entries in $\mathbf{p}_{i}$ add up to one and $\mathbf{v}_{i}{ }^{\mathrm{T}} \mathbf{p}_{i}=1$. These contributions represent the contribution of each size class to invasion and are therefore a way to ask which sizes contribute the most to invasion at any given point.

This article is protected by copyright. All rights reserved. 
Finally, we performed a sensitivity analysis of $\lambda_{i n v}$ with respect to the model parameters to assess how increases in competitive asymmetry between sizes promote coexistence or competitive exclusion through the different sizes. In Appendix S1, we show that the matrix approximation of this sensitivity for the IPM is:

$$
\frac{d \lambda_{i n v_{i}}}{d \theta^{\mathrm{T}}}{ }_{s}=\sum_{1}^{r}\left[\frac{d \lambda_{i n v_{i}}}{d \operatorname{vec}^{\mathrm{T}} \mathbf{K}_{i}} \circ\left(\frac{\partial v e c \mathbf{K}_{i}}{\partial \operatorname{vec}{ }^{\mathrm{T}} \boldsymbol{\alpha}_{i j}} \frac{d v e c \boldsymbol{\alpha}_{i j}}{d \theta^{\mathrm{T}}}+\sum_{V} \frac{\partial v e c \mathbf{K}_{i}}{\partial \mathbf{n}_{j}^{\mathrm{T}}} \frac{d \widetilde{\mathbf{n}}_{j}}{d v e c{ }^{\mathrm{T}} \boldsymbol{\alpha}_{j j}} \frac{d v e c \boldsymbol{\alpha}_{j j}}{d \theta^{\mathrm{T}}}\right)^{\mathrm{T}}\right]_{r, s},
$$

where $\theta$ is a scalar (or vector) that represents any of the parameters (or set of parameters) in the model. Importantly, $\mathbf{K}_{i}=\left.\mathbf{K}_{i}\right|_{\mathbf{n}_{i}=0, \mathbf{n}_{j}=\widetilde{\mathbf{n}}_{j}}$ is constructed at the single species equilibrium of the resident (species $j$ ) and the zero equilibrium of the invader (species $i$ ). The operator $\circ$, represents the element-by-element product and vec means to vector transform the matrix so that vec $\mathbf{K}_{i}=\left[k_{1,1}, \ldots, k_{r, 1}, k_{1,2}, \ldots, k_{r, 2}, \ldots, k_{1, s}, \ldots, k_{r, s}\right]^{\mathrm{T}}$, or so that columns, $s$, are stacked one on top of the other. The overall meaning of the derivative is to describe how small changes in the model parameter describing either competitive asymmetry or niche shifts influences invasion of species $i$. The derivative, $\frac{d \lambda_{i n v_{i}}}{d v e c{ }^{\mathrm{T}} \mathbf{K}_{i}}$, is the vector transformed sensitivity of the invasion growth rate of species $i$ to changes in the elements of the matrix approximation of the IPM projection kernel. The product of this derivative and the first term in parentheses describes the effect the parameter has on the invasion growth rate of the invader through interspecific competition. The product of $\frac{d \lambda_{i n v_{i}}}{d v e c{ }^{\mathrm{T}} \mathbf{K}_{i}}$ and the second term in parentheses describes how invasion of species $i$ changes through the effect that $\theta$ has on the equilibrium size distribution of the resident. When $\theta$ is a parameter of the invader, (e.g. $\left.\theta=\phi_{i}\right)$, then the second term is zero and the change in the invasion growth rate depends only on how the parameter change alters the interspecific interaction. When $\theta$ is a parameter of the resident (e.g. $\theta=\rho_{j}$ ) then both terms may be non-zero and the outcome is determined by a balance of 
the two terms, or the balance between the interspecific interactions and the intraspecific interactions of the resident. We present these size dependent sensitivities to show how small changes in the parameters influences invasion across the range of body sizes.

\section{RESULTS}

ECOLOGICAL SCENARIO 1: ONTOGENETIC NICHE SHIFTS WITH COMPETITIVE SYMMETRY

Coexistence between two species is not possible when interspecific competition and competition between sizes is symmetric $\left(\eta_{12}=0, \phi_{1}=0, \phi_{2}=0\right)$ and one species shifts its niche with size more than the other species (Figure 2A). Instead, the species that shifts its niche with ontogeny to a greater degree will exclude the other species (Figure 2A). When both species shift niches with size to an equal degree (diagonal line in Figure 2A), the invasion growth rate $\left(\lambda_{i n v}\right)$ of both species is equal to 1 and coexistence is neutral. Under the scenario where neither species shifts its niche with size (represented by the black dot in Figure 2A and B) the largest contributions towards the invasion growth rate of both species are for newborn individuals (Figure 2C and $\mathrm{D}$ ), but the majority of the mass of the distribution occurs in individuals above $\sim 10 \mathrm{~mm}$ SL (Figure $2 \mathrm{C}$ and D). Small changes to the degree of niche shift in species 2 decrease the invasion growth rate for species 1 across all sizes and increase the invasion growth rate for species 2 (Figure 2E). The later of these two changes is mostly a function of an increase in the contribution of larger sized individuals of species 2 escaping competition with smaller sizes of their own species and all sizes of species 1 (Figure 2E). In contrast, small changes in the asymmetry of species 1 increases its growth rate, mostly as a function of larger individuals (Figure $2 \mathrm{~F}$ ) and decreases the invasion growth rate of all sizes of species 2 (Figure $2 \mathrm{~F}$ ). Together, these results suggest that small increases in the size-dependent niche shift of species 2 should allow it to competitively exclude species 
1 and small changes in the size asymmetry of competition in species 1 should allow it to competitively exclude species 2 (because the signs of the sensitivities are opposite in each and the invasion growth rates for both species are equal to 1). As a whole, these results suggest that neither ontogenetic niche shifts alone nor small changes in either ontogenetic shifts or competitive ability are sufficient to promote coexistence.

\section{ECOLOGICAL SCENARIO 2: COMPETITIVE ASYMMETRY INCREASES WITH BODY SIZE IN ONE SPECIES}

Coexistence between the two species is possible when the competitive ability of one of the species increases with increasing body size and the other species shifts its niche with size to a larger degree $\left(\eta_{12}=0, \phi_{1}=0.02, \phi_{2}=0\right.$, Figure $\left.3 \mathrm{~A}\right)$. Assuming that species 1 does not shift its niche with body size, coexistence between the species is possible when species 2 shifts its niche to a moderate degree ( $\rho_{2}$ values between $\sim 0.0540$ and 0.1046 ; black dots in Figure 3A and B). At both of these boundary points, newborn individuals have the largest contribution to the invasion growth rate, but the majority of the mass of the distribution occurs in individuals above $\sim 10 \mathrm{~mm}$ SL (Figure $3 \mathrm{C}$ and D). At the first boundary, a small increase in the niche shift with size in species $2\left(\rho_{2}\right)$ is predicted to lead to coexistence - an effect that is dominated by the change associated with larger individuals of species 2 (Figure 3E). In contrast, small increases in the competitive ability of larger individuals of species $1\left(\phi_{1}\right)$ decreases $\lambda_{i n v_{2}}$ and prevents successful invasion of species 2 (negative derivative in Figure 3F); an effect that is spread more or less evenly across the range sizes. At the second boundary, where the invasion growth rate of species 1 becomes stable, small increases in the niche shift of species 2 decrease the invasion growth rate of species 1 more or less evenly across all sizes (Figure 3E). In contrast, small increases in size asymmetric competitive ability increase the invasion growth rate, an effect that is due mostly 
to larger individuals (Figure 3F). Overall, these results point towards coexistence emerging as a consequence of both ontogenetic changes in resource use and competitive asymmetries.

\section{ECOLOGICAL SCENARIO 3: COMPETITIVE ASYMMETRY INCREASES WITH SIZE}

\section{IN SPECIES 1 AND DECREASES WITH SIZE IN SPECIES 2}

A wider variety of outcomes is possible when small individuals of species $1(<15 \mathrm{~mm})$ are worse competitors than small individuals of species 2 and large individuals of species 1 $(>15 \mathrm{~mm})$ are better competitors than large individuals of species $2\left(\eta_{12}=0, \phi_{1}=\right.$ $0.06, \phi_{2}=-0.06, v=15 \mathrm{~mm}$; Figure 4). Under this scenario, when species 2 shifts its niche with body size, but species 1 does not (vertical axis in Figure 4A or Figure 5A) the region where coexistence is possible is similar to scenario 2: coexistence is possible under intermediate levels of ontogenetic niche shifts in species 2 (compare Figure 3A with Figure 4A). However, assuming that each species shifts its niche to an equal and large degree, then each species is able to competitively exclude the other (black areas in Figure 4A or Figure 5A). The species that persists will be the one that entered the habitat first (priority effect). Below we analyze the model at transition points between the regions in which these outcomes occur and the regions of competitive exclusion.

\section{Region of coexistence}

The region of coexistence in scenario 3 (Figure 4A and B) is similar to that in scenario 2 (Figure $3 \mathrm{~A}$ and B). In both scenarios, the invasion growth rates of both species at the boundary have the largest contributions from newborns, but the majority of the mass of the distributions occurs in the sizes greater than $\sim 10 \mathrm{~mm}$ SL (Figure $4 \mathrm{C}$ and D). Small increases in the degree of size based niche shift of species 2 are also similar between the two scenarios with large individuals of species 2 contributing the most towards the increase in the 
invasion growth rate and more or less even contribution of all sizes of species 1 contributing the decrease in the invasion growth rate (Figure 4E). In contrast to scenario 2, small increases in competitive asymmetry with size in species 1 decreases the invasion growth rate of species 1 through smaller individuals and increases the invasion growth rate through larger individuals (Figure 4F). The net change (sum of derivatives for species 1 in Figure 4F) through all individuals will decrease the invasion growth rate and hence lead to competitive exclusion of species 1 by species 2 . Also in contrast to scenario 2, small increases in sizebased asymmetries of species 1 increase the invasion growth rate of species 2 , but these effects are very small across all sizes (Figure 4F). Overall, while the dynamics at the boundary between species 1 competitively excluding species 2 and coexistence appear similar between the two scenarios, the dynamics at the boundary between coexistence and competitive exclusion by species 2 are opposite. In scenario 2, increasing size asymmetry of species 1 expanded the boundary while in this scenario it caused the boundary to contract in favor of competitive exclusion of the other species.

\section{Region of priority effects}

A region of priority effects appears when both species shift their niches with size to an equal degree and when the shift is extreme enough such that larger individuals compete very little with smaller individuals (Figure 5A). Moving from the origin along a diagonal where the niche shift in species 1 and 2 are equal (Figure 5A and B), the region of priority effects emerges when both species shift their niches to a large degree $(\rho \sim 0.1201)$. At this boundary point, smallest individuals have the largest contribution to the invasion growth rate and the contribution of larger individuals progressively decreases with increasing size (Figure 5D). Moving further along the diagonal, the zero equilibrium of species 2 then becomes unstable at the second boundary along the diagonal $(\rho \sim 0.1587)$ and species 2 can 
competitively exclude species 1 . At this boundary point, and in contrast to all other boundaries, larger individuals have the largest contribution to the invasion growth rate (Figure 5C). Also in contrast to other scenarios, small increases in the degree of niche shift of individuals of species 2 increase the invasion growth rate of species mostly through effects on medium sized individuals (Figure 5E). Small increases in the niche shift of species 2 decrease the invasion growth rate of species 1 at the first boundary-an effect that is fairly evenly distributed across sizes. Small increases to the degree of size asymmetry in species 1 decrease the invasion growth rate of species 1 through small individuals and increase it through larger individuals.

\section{DISCUSSION}

We developed a discrete time demographic model of two interacting size-structured species where both the niche and competitive ability can change through ontogeny. We asked whether complex life histories in the form of continuous changes in the niche and competitive asymmetries facilitate or impede coexistence. Our results suggest that when both species can shift their niche with increasing body size and competition for resources among the sizes is symmetric, then the species that shifts its niche to a greater degree with ontogeny will competitively exclude the other species (Figure 2A). When competitive ability increases with increasing body size, then the two species can coexist when the better competitor shifts its niche with body size to a lesser degree than the weaker competitior (Figure 3A). When both species shift their niches with size, but each is a better competitor on resources used by smaller or larger individuals, then the model predicts an alternative stable state where one species can exclude the other depending on which species enters the habitat first (Figure 4A and 5A). In all cases, the region of coexistence was small compared to the regions predicting 
competitive exclusion. The region of coexistence was largest when one species shifts its niche with size and the other does not; the region of coexistence shrank as the initially nonshifting species shifted its niche with size.

A basic tenet of species coexistence theory is that coexistence is posssible if the total effect of intra-specific competition across the life stages on population growth rate is greater than the total effect of interspecific competition on the population growth rate of both species. This tenet holds true even in the cases presented here where the life history, niche shifts and competitive asymmetry changes continuously throughout life. This can be seen in the context of the model because for $\lambda_{i n v_{i}}$ to be greater than 1 , the change in $\lambda$ associated with competing with only the other species $\left(\Delta \lambda_{i j}=\left.\lambda_{i}\right|_{n_{i}=0, n_{j}=0}-\left.\lambda_{i}\right|_{n_{i}=0, n_{j}=\tilde{n}_{j}}\right)$ must be less than the change in $\lambda$ associated with competiting only with individuals of the same species $\left(\Delta \lambda_{i i}=\left.\lambda_{i}\right|_{n_{i}=0, n_{j}=0}-\left.\lambda_{i}\right|_{n_{i}=\tilde{n}_{i}, n_{j}=0}\right)$. In simple Lotka-Volterra models, this generally requires some niche differentiation between the two species. In two-species, two-stage consumer resources models the result depends on how niche differentiation and competitive asymmetries interact with the life history to determine which stage limits or regulates the populations. Coexistence will occur if these factors interact such that the two competiting populations are limited by different life stages and each is a better competitor in the stage that limits the population (e.g. Loreau and Ebenhoh 1994). In multi-stage or continuous stage populations, the life history, niche shifts and competitive asymetries can interact to produce a variety of regulation patterns across the life cycle. The results of the sensitivity analyses suggest that, sometimes, change in predictions regarding coexistence will arise from changes that occur at the extremes of the life cycle (as in Figure 2E, species 2), sometimes from changes spread evenly across the life cycle (as in Figure 2E, species 1), or sometimes in the middle of the life cycle (as in Figure 5E, species 2). The framework we propose here should allow a development of coexistence theory in multi-stage or continuous stage populations. 
The model we present illustrates the variety of outcomes that are possible when both the niche and competitive ability change continuously through ontogeny. We used body size to illustrate the model because it is very often the trait that determines changes in the niche and competitive outcomes. In practice, any continuous trait could be used. In addition, different components of the interaction surface can be eliminated or modified to accommodate a wide variety of niche changes. Other types of continuous ontogenetic niche changes could be included. For example, instead of the niche shifting its mean position with increases in size, the niche breadth could be modelled as a function of size. This would allow the niches of larger individuals to overlap completely with smaller individuals, but also to occupy some niche regions where they do not compete witih smaller individuals. In other cases, no traitbased competitive asymmetries may exist and so the parameter governing competitive size asymmetries may be safely assumed to be zero.

A key strength of our approach is that our models can be parameterised with data that are routinely collected by ecologists. Fitting the model to data means that researchers can use the model to interpret the results of experiments in a way that integrates all the information on changes in demographic rates, as opposed making inferences based on examining one demographic rate at a time. Our work, as well as that championed by Adler et al. (2010), illustrates this point; their paper and ours combine a model of demographic rate changes with field data to ask whether competition has a role in structuring biological communities. Our hope is that the framework outlined here will give others the tools to answer questions about coexistence in a wider variety of systems.

This article is protected by copyright. All rights reserved. 


\section{ACKNOWLEDGMENTS}

RDB, JT, and TC conceived the ideas presented. RDB developed and analyzed the models with input from JT and TC. RDB wrote the first draft of the manuscript and all authors contributed substantially to the final version. We wish to thank Yuridia Reynoso and the numerous field and laboratory technicians that helped with the mesocosm experiments and dissection of the guppies. We also wish to thank Mark Rees, Dylan Childs and Shripad Tuljapurkar for discussions regarding competitive asymmetries. The mesocosm and field research was originally funded by USA NSF research grants (EF0623632, 9419823) and the model development and analyses presented here were funded by a UK NERC grant (ATR00350) and a USA NSF grant (DEB 1556884). TC also acknowledges support from an ERC advanced grant (LEED, number 249872).

\section{Literature Cited}

Ackleh, A. S., and R. A. Chiquet. 2011. Competitive exclusion in a discrete juvenile-adult model with continuous and seasonal reproduction. Journal of Difference Equations and Applications 17:955-975.

Adler, P. B., S. P. Ellner, and J. M. Levine. 2010. Coexistence of perennial plants: an embarrassment of niches. Ecology Letters 13:1019-1029.

Bassar, R. D., D. Z. Childs, M. Rees, S. Tuljapurkar, D. N. Reznick, and T. Coulson. 2016. The effects of asymmetric competition on the life history of Trinidadian guppies. Ecology Letters 19:268-278.

Bassar, R. D., A. López-Sepulcre, D. N. Reznick, and J. Travis. 2013. Experimental evidence for density-dependent regulation and selection on Trinidadian guppy life histories. The American Naturalist 181:25-38.

This article is protected by copyright. All rights reserved. 
Briones, J. C., C.-H. Tsai, T. Nakazawa, Y. Sakai, R. D. S. Papa, C.-h. Hsieh, and N. Okuda. 2012. Long-term changes in the diet of Gymnogobius isaza from Lake Biwa, Japan: effects of body size and environmental prey availability. PLoS One 7:e53167.

Caswell, H. 2001. Matrix Population Models. 2nd edition. Sinauer Ass., Sunderland, Ma.

Corbet, P. 1980. Biology of Odonata. Annual Review of Entomology 25:189-217.

Coulson, T. 2012. Integral projections models, their construction and use in posing hypotheses in ecology. Oikos 121:1337-1350.

Coulson, T., S. Tuljapurkar, and D. Z. Childs. 2010. Using evolutionary demography to link life history theory, quantitative genetics and population ecology. Journal of Animal Ecology 79:1226-1240.

de Roos, A. M., and L. Persson. 2013. Population and community ecology of ontogenetic development. Princeton University Press, Princeton, New Jersey.

Easterling, M. R., S. P. Ellner, and P. M. Dixon. 2000. Size-specific sensitivity: applying a new structured population model. Ecology 81:694-708.

Haefner, J. W., and J. L. Edson. 1984. Community invasion by complex life cycles. Journal of Theoretical Biology 108:377-404.

Loreau, M., and W. Ebenhoh. 1994. Competitive exclusion and coexistence of species with complex life cycles. Theoretical Population Biology 46:58-77.

Macarthur, R., and R. Levins. 1967. The limiting similarity, convergence, and divergence of coexisting species. The American Naturalist 101:377-385.

Miller, T. E. X., and V. H. W. Rudolf. 2011. Thinking inside the box: community-level consequences of stage-structured populations. Trends in Ecology \& Evolution 26:457466.

This article is protected by copyright. All rights reserved. 
Moll, J., xa, D, J. Brown, xa, S, F. M. Associate Editor: William, and L. D. Editor: Donald. 2008. Competition and Coexistence with Multiple Life\&\#x2010;History Stages. The American Naturalist 171:839-843.

Nakazawa, T. 2015. Ontogenetic niche shifts matter in community ecology: a review and future perspectives. Population Ecology 57:347-354.

Rees, M., D. Z. Childs, and S. P. Ellner. 2014. Building integral projection models: a user's guide. Journal of Animal Ecology 83:528-545.

Reñones, O., N. V. C. Polunin, and R. Goni. 2002. Size related dietary shifts of Epinephelus marginatus in a western Mediterranean littoral ecosystem: an isotope and stomach content analysis. Journal of Fish Biology 61:122-137.

Roth, G., P. L. Salceanu, and S. J. Schrieber. In press. Robust permanence for ecological maps. SIAM Journal or Mathematical Analysis.

Wallace, K. M., and A. J. Leslie. 2008. Diet of the Nile Crocodile (Crocodylus niloticus) in the Okavango Delta, Botswana. Journal of Herpetology 42:361-368.

Werner, E., and J. F. Gilliam. 1984. The ontogenetic niche and the species interactions in size-structured populations. Annual Review of Ecology and Systematics 15:393-425.

Williams, J. L., T. E. X. Miller, and S. P. Ellner. 2012. Avoiding unintentional eviction from integral projection models. Ecology 93:2008-2014.

This article is protected by copyright. All rights reserved. 
Table 1. Parameters definitions, vital rate functions and matrix equivalents.

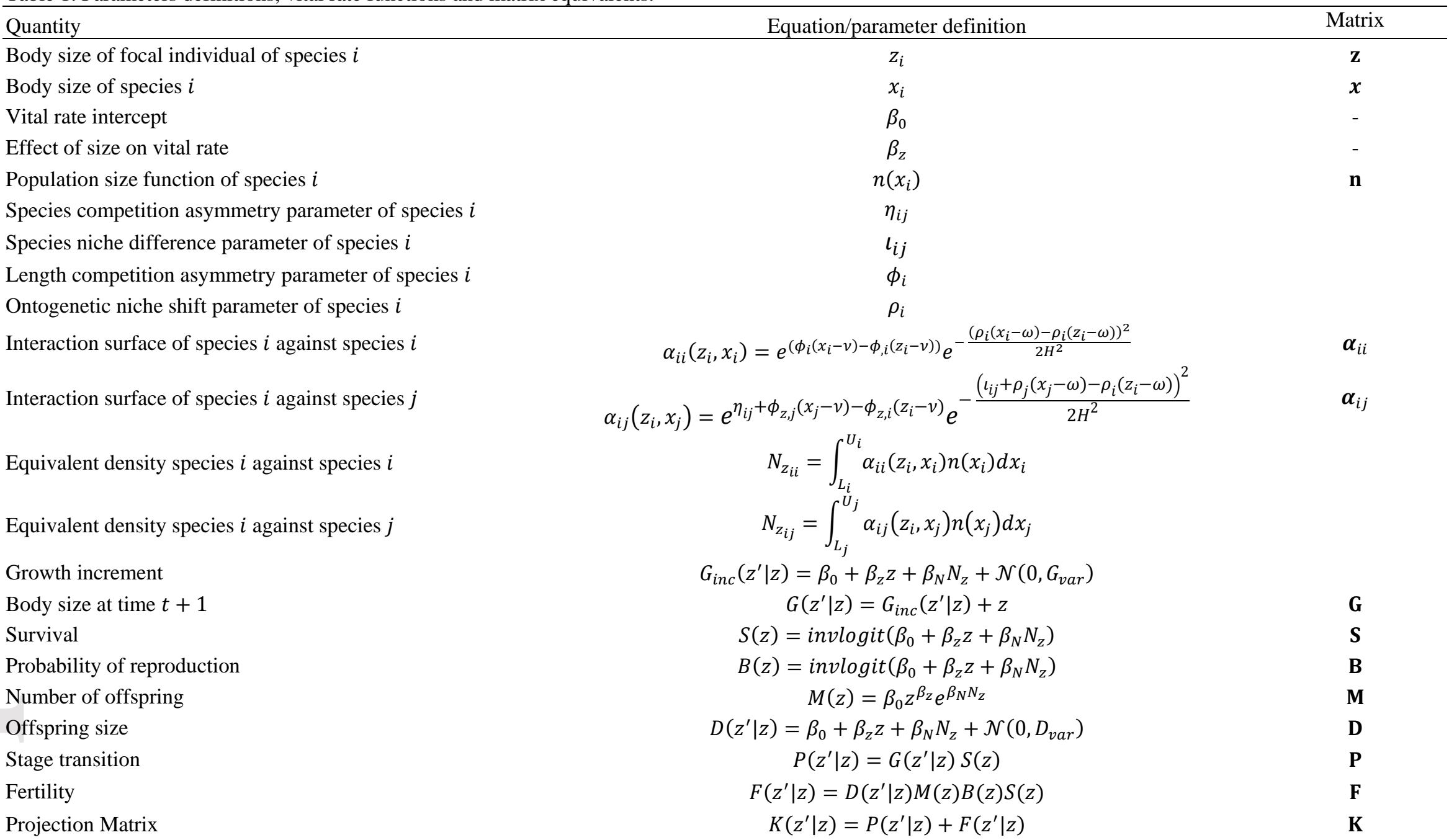

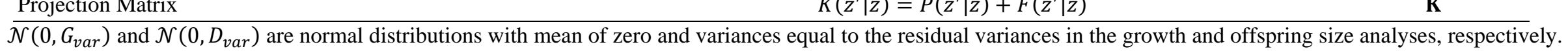

This article is protected by copyright. All rights reserved. 


\section{FIGURE LEGENDS}

Figure 1. Interaction surfaces for two species model when newborns of species $j$ share a niche with all individuals of species $i$, but larger individuals of species $j$ do not $\left(\iota_{i j}=0, \rho_{i}=\right.$ $0, \rho_{j}=0.15, \omega=6.5 \mathrm{~mm}$ ). Columns give the interaction surfaces for the within and between species interaction surfaces for species $i$ and species $j$, respectively. The first row presents the shape of the interaction surfaces when all individuals of both species are equally competitive (symmetric competition; $\eta_{i j}=0, \phi_{i}=0, \phi_{j}=0$ ). The second row presents the shape of the interaction surfaces when competitive ability increases with body length in species $i$ (length asymmetry; $\left.\eta_{i j}=0, \phi_{i}=0.04, \phi_{j}=0\right)$. Yellow space represents regions where the interaction surface is near or equal to 0. Orange areas are where the interaction surface is equal to 1 , as in the upper left panel. Red areas are regions where the interactions surfaces are greater than 1.

Figure 2. Results of scenario 1 where we examine the coexistence of two species with the same life history and where all individuals of both are equally competitive (competitive symmetry; $\left.\eta_{i j}=0, \phi_{1}=0, \phi_{2}=0\right)$. Newborns of both species share a niche $(\omega=$ $6.5 \mathrm{~mm}, \iota_{i j}=0$ ) and larger individuals of both species can shift to varying degrees with increased body size. Panels represent A) coexistence plot, B) invasion growth rate when species 1 has no ontogenetic niche shift and species 2 shift to varying degrees, C and D) the contribution of individuals of each size to the invasion growth rate $\left(\lambda_{i n v}\right)$ for the boundary points for species 2 and 1, respectively, and $\mathrm{E}$ and $\mathrm{F}$ ) the sensitivity of the invasion growth rate to changes in niche shifts of species 2 and increased competitive ability of species 1 at the boundary for each species. In panel A, white regions are combinations of ontogenetic niche shifts where species 1 is predicted to exclude species 2. Dark grey regions are combinations of ontogenetic niche shifts where species 2 is predicted to exclude species 1 . 
In panel $\mathrm{B}$, regions of the functions where $\lambda_{i n v}<1$ indicate where one or both zero equilibria are stable and mutual invasion is not possible. Sections where the $\lambda_{i n v}>1$ represent regions where the zero equilibria are unstable and mutual invasion, and therefore coexistence, is possible. For panels $\mathrm{E}$ and $\mathrm{F}$, the sensitivities are measured at the zero equilibrium of the invader when $\lambda_{\text {inv }}=1$ given by the points in panels $\mathrm{A}$ and $\mathrm{B}$. The distribution of these sensitivities describes how different sizes contribute to invasion.

Figure 3. Results of scenario 2 where we examine the coexistence of two species with the same life history and where the larger individuals of species 1 are better competitors $\left(\boldsymbol{\eta}_{\boldsymbol{i j}}=\mathbf{0}\right.$, $\left.\boldsymbol{\phi}_{1}=\mathbf{0 . 0 2}, \boldsymbol{\phi}_{2}=\mathbf{0}\right)$. Newborns of both species share a niche $\left(\boldsymbol{\omega}=\mathbf{6 . 5} \mathbf{m m}, \boldsymbol{\iota}_{\boldsymbol{i j}}=\mathbf{0}\right)$ and larger individuals of both species can shift to varying degrees with increased body size. Panel descriptions are the same as those in Figure 2. In panel A, white regions are combinations of ontogenetic niche shifts where species 1 is predicted to exclude species 2. Dark grey regions are combinations of ontogenetic niche shifts where species 2 is predicted to exclude species 1. Light grey regions are combinations of ontogenetic niche shifts where the two species are predicted to coexist (labelled "Coex").

Figure 4. Results of scenario 3 where we examine the coexistence of two species with the same life history where both species can shift or not, but each is a better competitor at different sizes - i.e. where competitive ability increases with size in species 1 and decreases with increasing size in species $2\left(\boldsymbol{\eta}_{12}=\mathbf{0}, \boldsymbol{\phi}_{\mathbf{1}}=\mathbf{0 . 0 6}, \boldsymbol{\phi}_{2}=-\mathbf{0 . 0 6}, \boldsymbol{v}=15 \mathrm{~mm}\right)$. As in other scenarios, newborns of both species share a niche $\left(\boldsymbol{\omega}=\mathbf{6 . 5} \mathbf{m m}, \boldsymbol{\iota}_{\boldsymbol{i j}}=\mathbf{0}\right)$ and larger individuals of both species can shift to varying degrees with increased body size. The parameter value $\boldsymbol{v}=\mathbf{1 5} \mathbf{~} \mathbf{m m}$ means that the two species are equal in their competitive ability at a size of $15 \mathrm{~mm}$. Panel descriptions are the same as those in Figure 2. In panel A, white regions are combinations of ontogenetic niche shifts values where species 1 is predicted to 
exclude species 2. Dark grey regions are combinations of ontogenetic niche shifts where species 2 is predicted to exclude species 1. Light grey regions are combinations of ontogenetic niche shifts where the two species are predicted to coexist (labelled "Coex"). Black regions are combinations of ontogenetic niche shifts with priority effects (labelled "Prior").

Figure 5. Results of scenario 3 where we examine the coexistence of two species with the same life history where both species can shift or not, but each is a better competitor at different sizes - i.e. where competitive ability increases with size in species 1 and decreases with increasing size in species $2\left(\boldsymbol{\eta}_{12}=0, \phi_{1}=0.06, \phi_{2}=-0.06, \boldsymbol{v}=15 \mathrm{~mm}\right)$. As in other scenarios, newborns of both species share a niche $\left(\boldsymbol{\omega}=\mathbf{6 . 5} \mathbf{m m}, \boldsymbol{\iota}_{\boldsymbol{i j}}=\mathbf{0}\right)$ and larger individuals of both species can shift to varying degrees with increased body size. The parameter value $\boldsymbol{v}=\mathbf{1 5} \mathbf{~} \mathbf{m m}$ means that the two species are equal in their competitive ability at a size of $15 \mathrm{~mm}$. Panel descriptions are the same as those in Figure 2. In panel A, white regions are combinations of ontogenetic niche shifts values where species 1 is predicted to exclude species 2. Dark grey regions are combinations of ontogenetic niche shifts where species 2 is predicted to exclude species 1. Light grey regions are combinations of ontogenetic niche shifts where the two species are predicted to coexist (labelled "Coex"). Black regions are combinations of ontogenetic niche shifts with priority effects (labelled "Prior").

This article is protected by copyright. All rights reserved. 


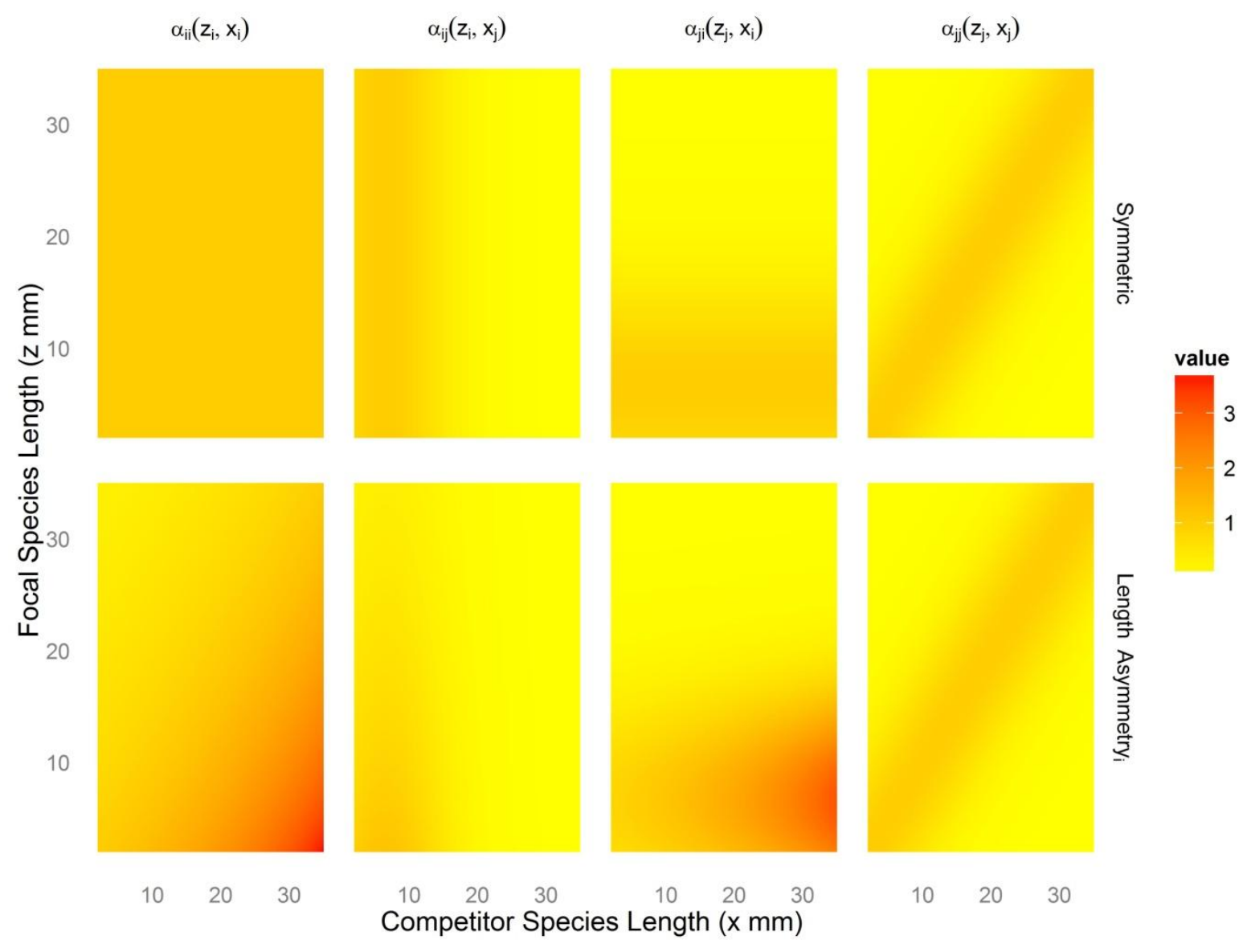

This article is protected by copyright. All rights reserved. 

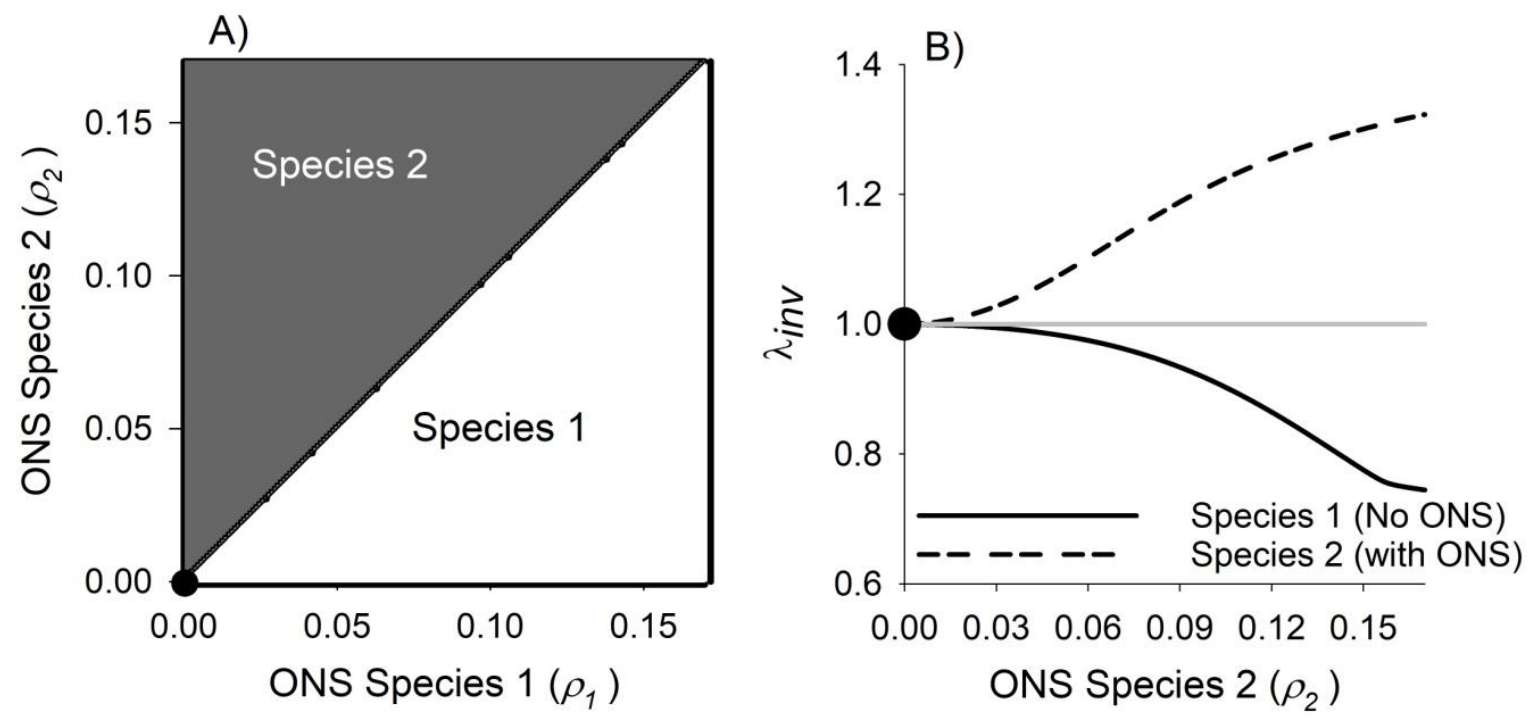

C)
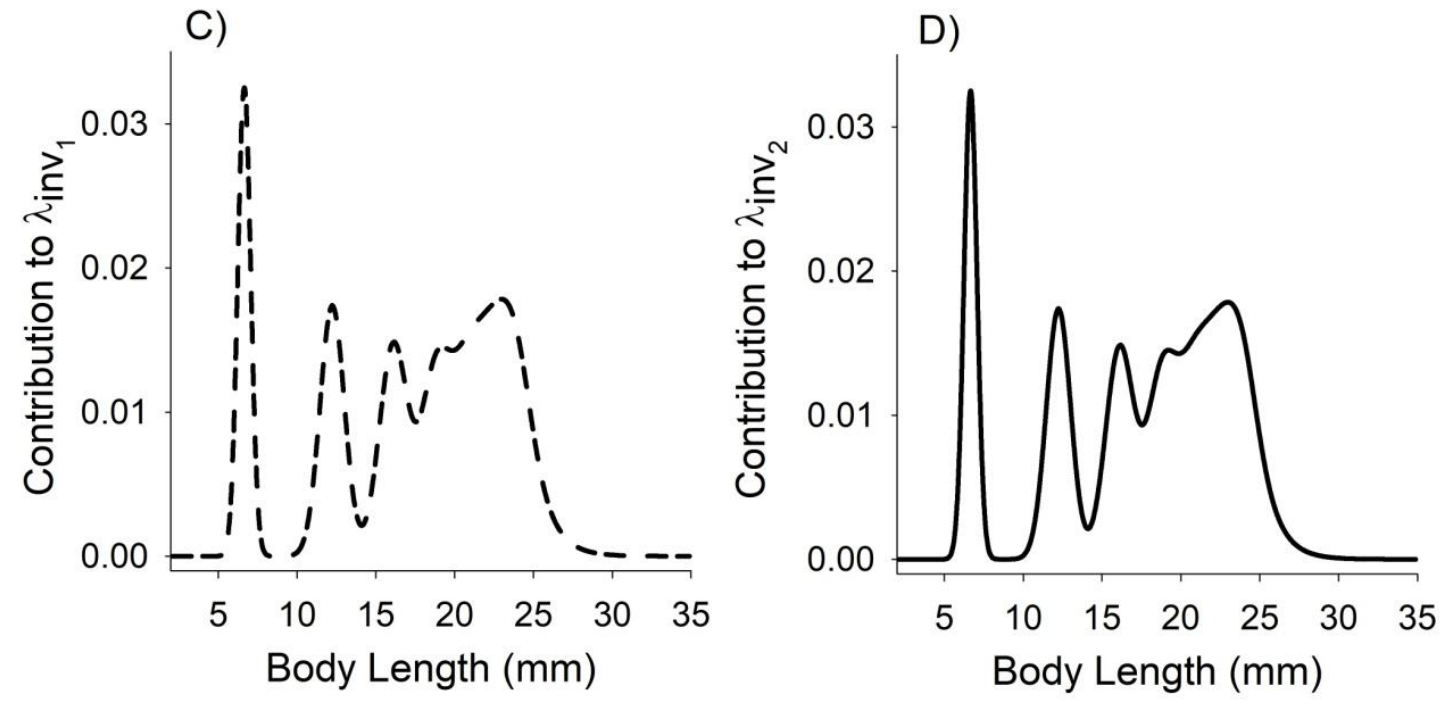

E)
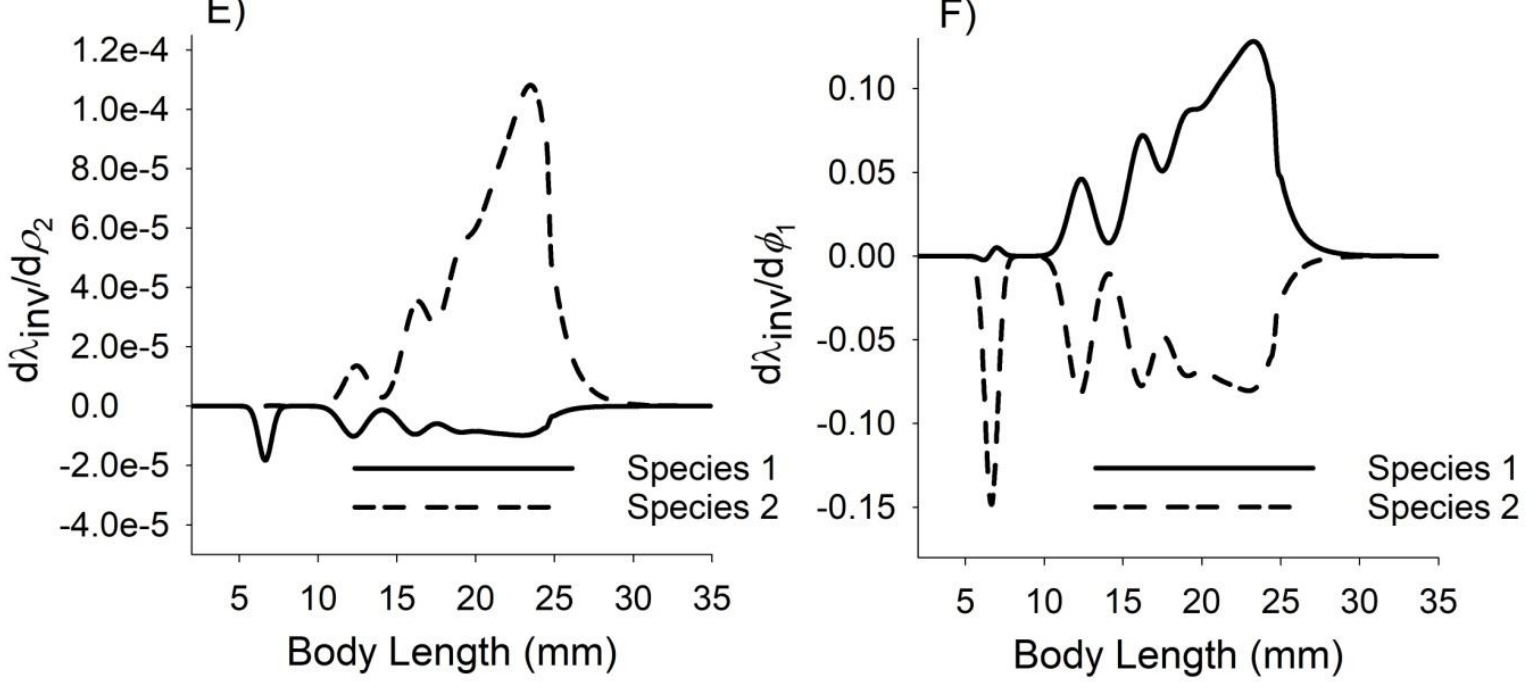

This article is protected by copyright. All rights reserved. 

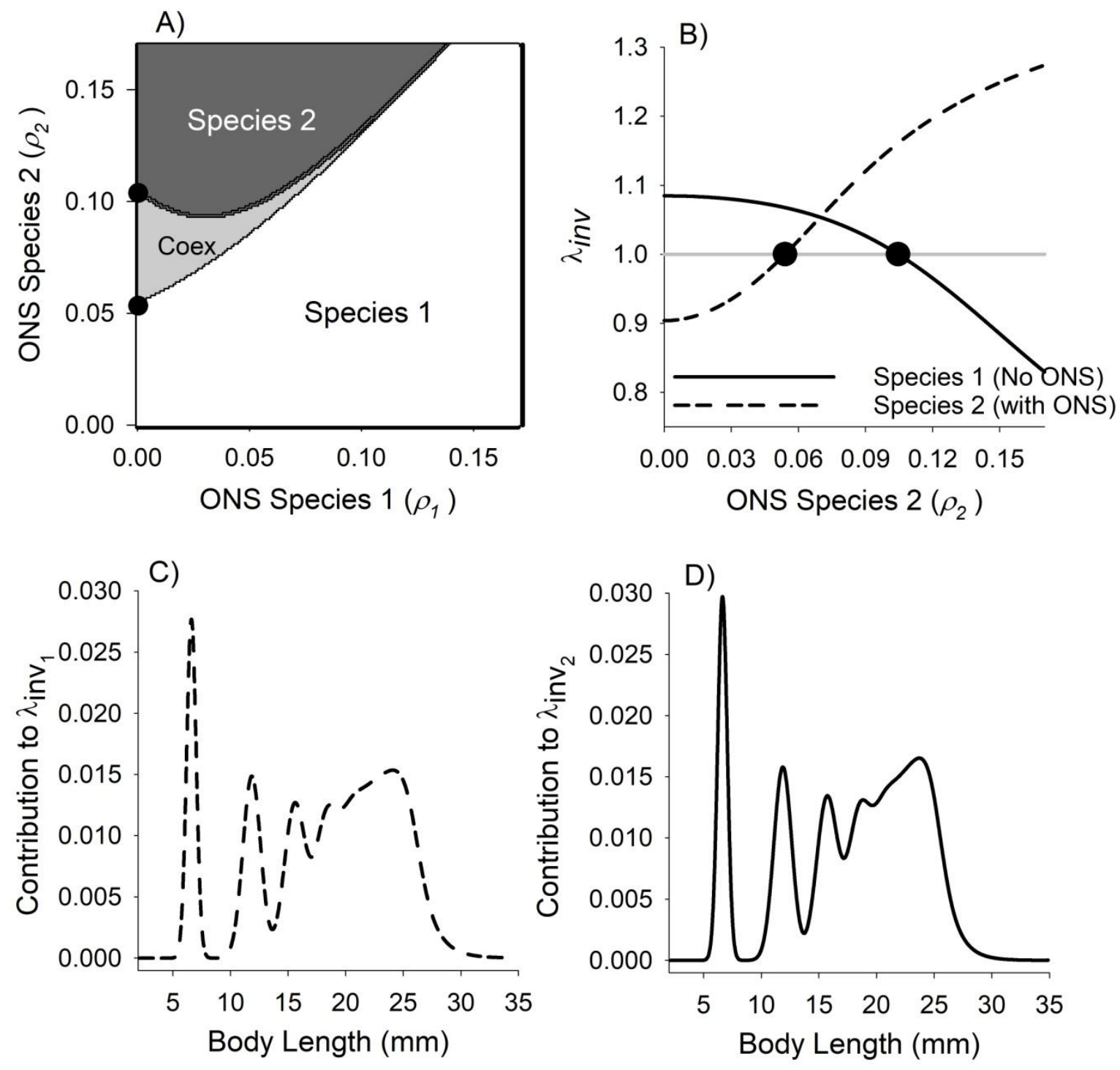

E)
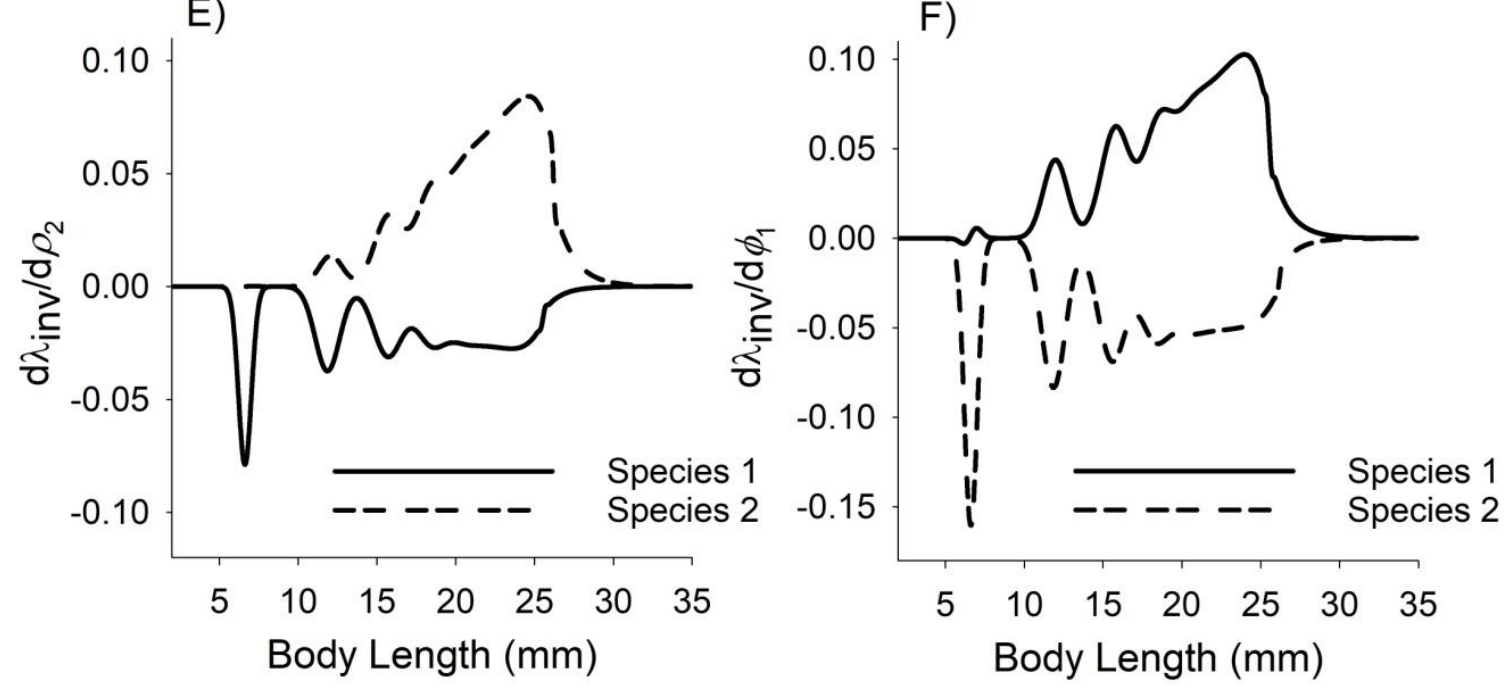

This article is protected by copyright. All rights reserved. 

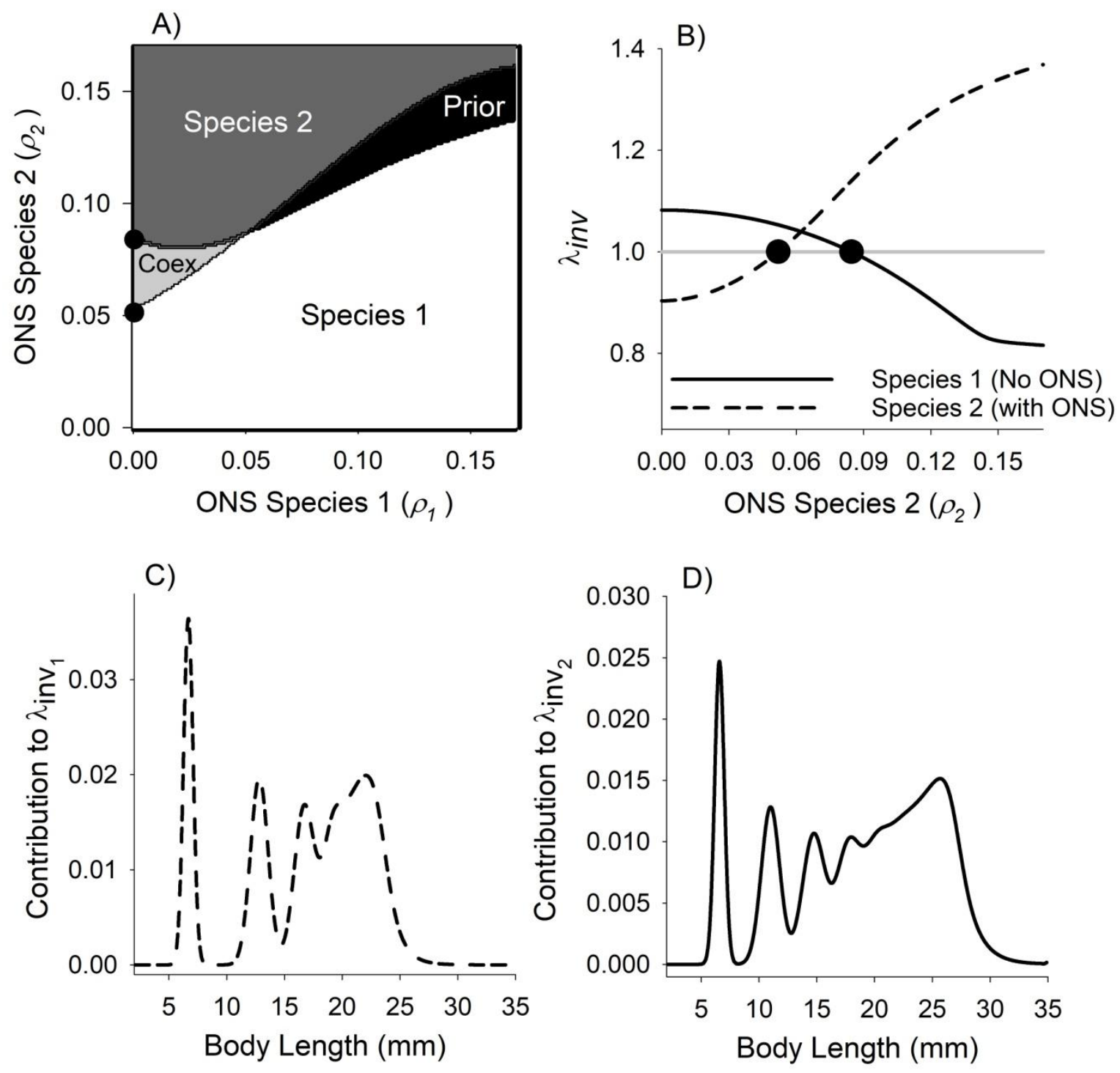

E)
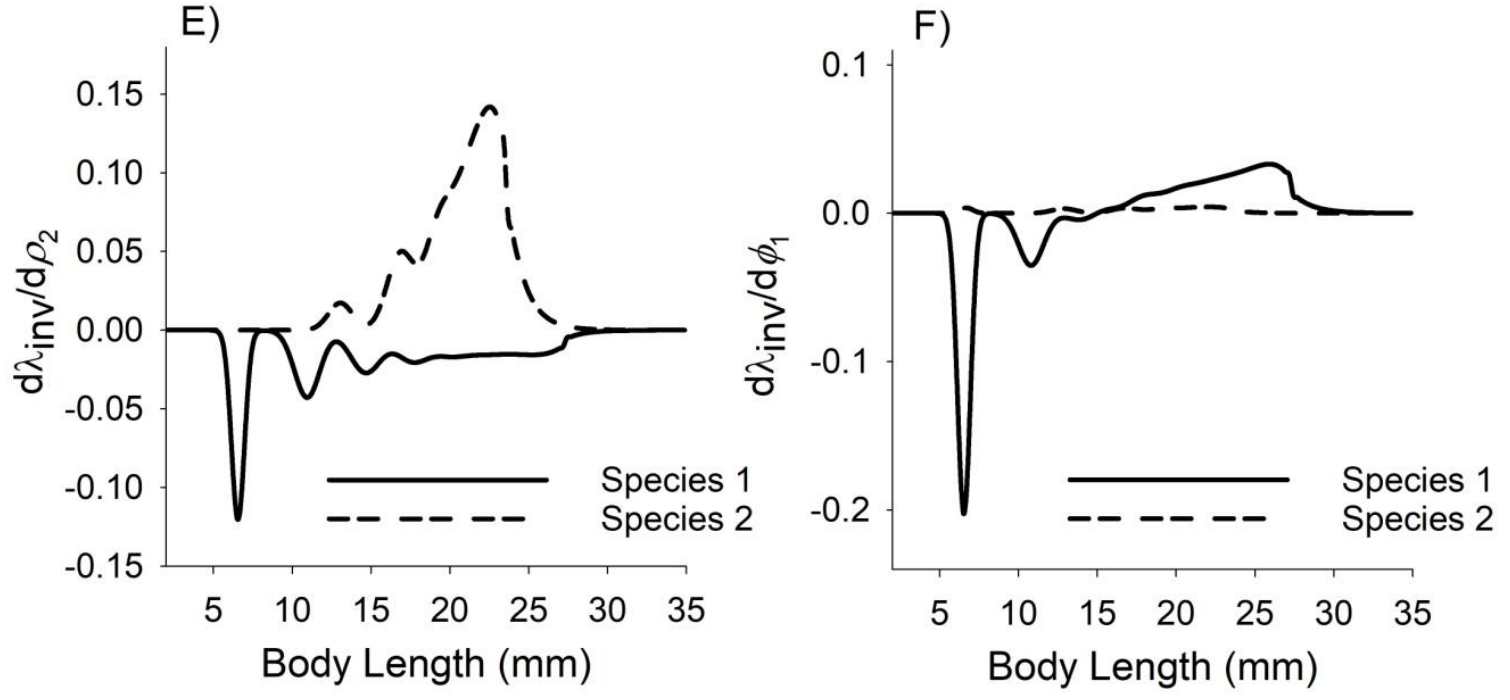

This article is protected by copyright. All rights reserved. 
A)

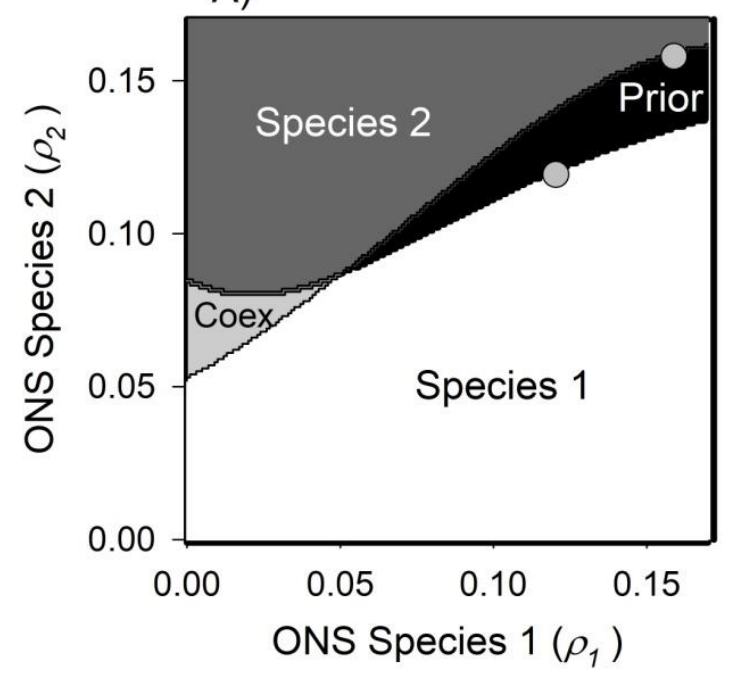

C)

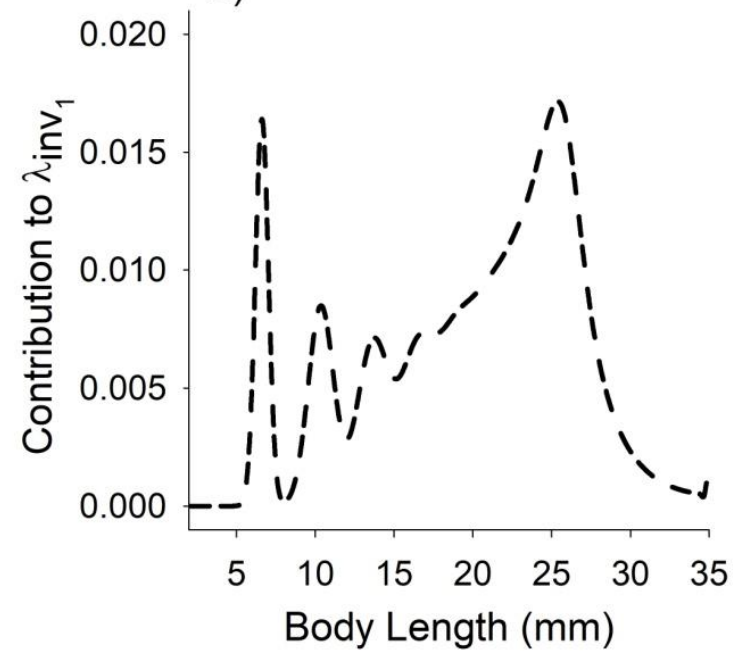

E)

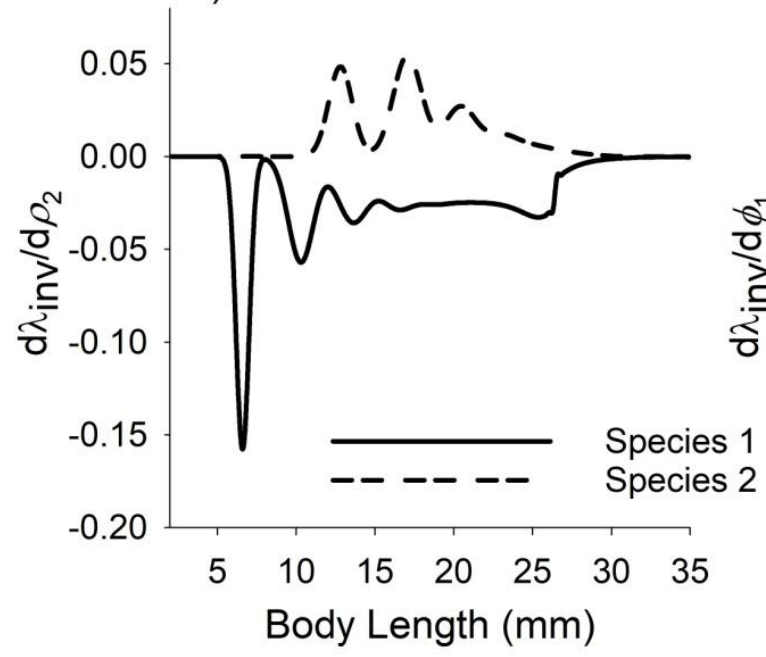

B)

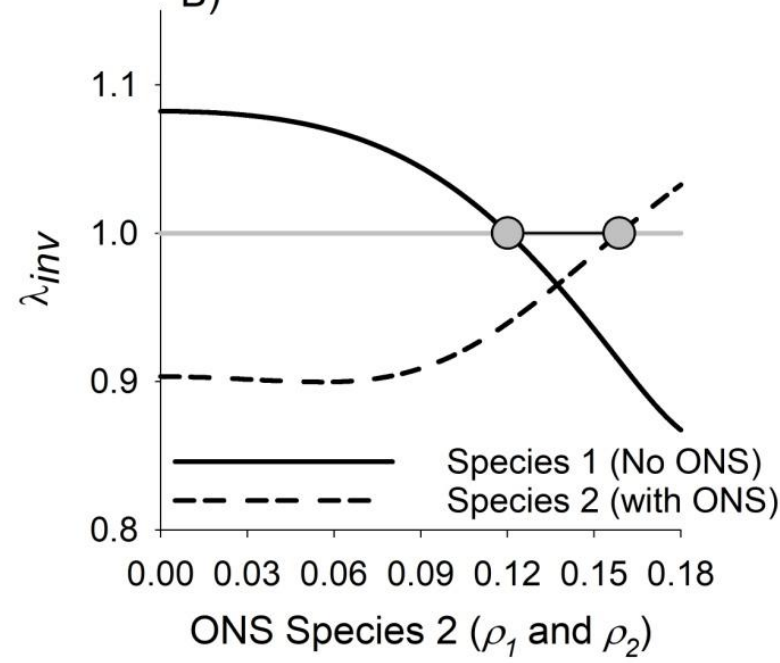

D)

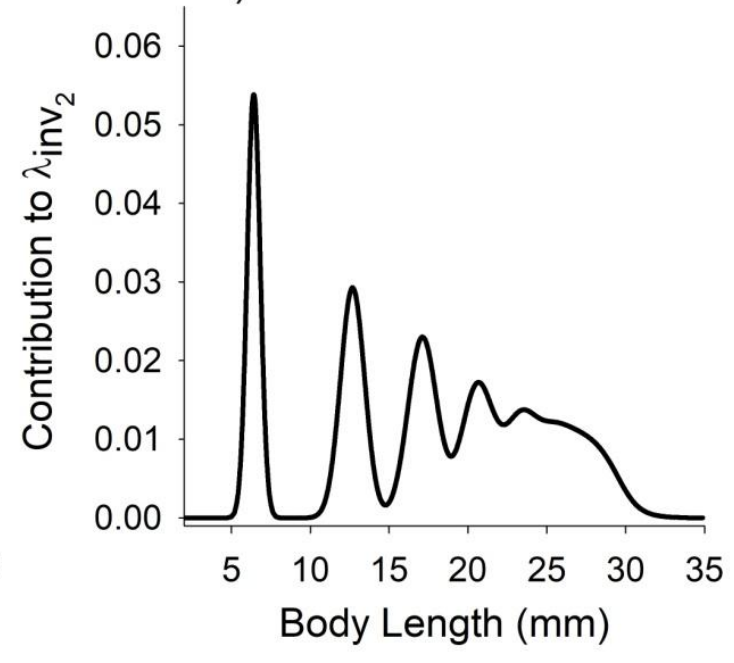

F)

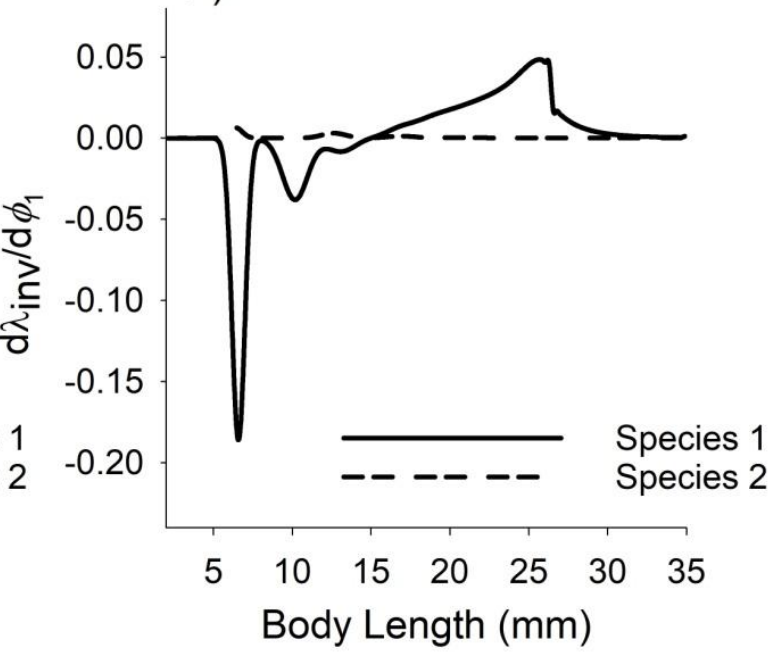

This article is protected by copyright. All rights reserved. 